Literature Reviews

\title{
Review of Periprosthetic Infection of Brucellosis with Presentation of a Case Report
}

\author{
${ }^{1}$ Yuri Klassov, ${ }^{2}$ Talya Marina Klassov, ${ }^{3}$ Odelya Peretz and ${ }^{4}$ Vadim Benkovich \\ ${ }^{1,4}$ Depertment of Orthopaedics and Arthroplasty, \\ Soroka University Medical Center, Itzhak Reger st. 151, 84101, Beer-Sheva, Israel \\ ${ }^{23}$ Soroka University Medical Center, Itzhak Reger st. 151, 84101, Beer-Sheva, Israel
}

Article history

Received: 28-04-2016

Revised: 02-08-2016

Accepted: 09-08-2016

Corresponding Author:

Yuri Klassov

Depertment of Orthopaedics

and Arthroplasty, Soroka

University Medical Center,

Itzhak Reger st. 151, 84101,

Beer-Sheva, Israel

Email: yuriklassov@gmail.com

\begin{abstract}
Nowadays there is an increase in number of arthroplasties performed worldwide. There are evidences that this trend will continue to rise on a yearly basis. Presence of a foreign body implant increases the risk for infection regardless the most meticulous infection control. Brucellosis is the most frequently encountered worldwide zoonotic disease. It is most often acquired from unpasteurized milk and dairy products. Although it can affect any organ in the human body, osteoarticular involvement is the most common complication seen almost in half of brucellosis infections. In this study we will present a review of periprosthetic infections in general and later we will focus on brucellosis periprosthetic infection, with presentation of a case that we investigated treated and latter followed in our hospital. There were only 19 cases reported worldwide in the English literature and this is the 4th case reported in Israel. To our knowledge this is the only case were the bacteria could not been eradicated but only suppressed while the patient would have to be treated with antibiotics and followed for life. We reviewed all the previous cases and then summarized treatment and follow up with discussion about the most appropriate investigation and treatment protocol. Although Brucellosis is a rare disease, while treating it and following our patient we realized how important it is to discuss and review this infection in order to help our patients as soon as possible in order to allow them to return to their daily living with minimal disabilities.
\end{abstract}

Keywords: Knee, Arthroplasty, Periprostetic Infections, Brucella, Total Knee Replacement

\section{Introduction}

Nearly one million Total Hip Arthroplasties (THAs) or Total Knee Arthroplasties (TKAs) are performed in the United States each year. It is estimated that by the year of 2030 more than four million THAs or TKAs will be performed annually only in the United States (Widmer, 2001).

Contrary to other internal fixation devices that are used in orthopedic surgeries today, which could be removed after fracture healing occurred, prosthetic joints are kept in the body as long as their function remains full and painless. The presence of an implant increases the risk for an infection because its local susceptibility for bacterial adherence is high (Zimmerli et al., 2004). Thus, even with the most meticulous infection control, implant infection does occur. The rate of Prosthetic Joint Infection (PJI) in most centers ranges between 0.5 to $1.0 \%$ for hip replacement, 0.5 to $2 \%$ for knee replacement (Widmer,
2001; Anonymous, 1995). Risk factors that have been found to be associated with PJI are superficial surgical site infection, prior joint arthroplasty, systemic or joint malignancy, rheumatic diseases and immunosuppressive therapy, diabetes mellitus, obesity (body mass index $>$ 30), postoperative bleeding hematoma formation, prolonged duration of surgery, post operative hyperglycemia and a "net state of polymorbidity" of a patient (e.g., number of comorbidities and physical status) (Kurtz et al., 2010; Poss et al., 1995; Jamsen et al., 2012). Timing of infection tells us a lot about the identity of the infecting organism. Early and late onset infections are most often due to relatively virulent pathogens, such as S. aureus or gram-negative bacilli. Delayed onset infections are often due to less virulent pathogens, such as Propionibacterium species and coagulase negative staphylococci (Elie and Larry, 2015). We want to present the distribution of pathogens as we have reviewed in English literature (Berbari et al., 1998): 
- Staphylococcus aureus-22\%

- Coagulase-negative staphylococci-19\%

- Mixed infection

- Beta hemolytic streptococci-9\%

- Aerobic gram-negative bacilli-8\%

- Anaerobes-6\%

- No organisms detected- $12 \%$

- Other organisms-5\%

Brucellosis is the most frequently encountered world wide zoonotic disease, which can be acquired from sheep, goat, cattle and swine (Bosilkowski et al., 2009). The disease is most frequently transmitted from unpasteurized milk and dairy products. Other routes of transmission to humans is by the way of direct contact with infected animal or their secretions through bruises and laceration on the skin, inhalation of infected aerosol, conjuctival inoculation, human to human transmission, congenital and sexual transmissions were reported as well (Doganay and Aygen, 2003; Mesner et al., 2007), together with that it is one of the most common laboratory acquired infections and it is classified as category B biological weapon by the Centers for Disease Control and Prevention (CDC) owing to the ease of transmission (Singh, 2009).

Brucella spp. is a small, gram negative, non motile, non spore forming, intracellular-reproducing, aerobic coccobacilli. Four species are pathogenic to humans: Brucella abortus, B.melitensis, B.suis and B.canis. The bacterium survives for 6 weeks at $4^{\circ} \mathrm{C}$ in cream, 30 days in ice cream and 15 to 100 days in fresh cheese. The bacterium is sensitive to ionized radiation, disinfectants, boiling or pasteurizing the milk also if the milk goes sour or lactic fermentation occurs (Cem Gul and Erdem, 2009). The incidence of brucellosis across the world varies from less than 0.03 to 160 per 100,000 people (Pappas et al., 2006). Brucellosis is most commonly seen in the Mediterranean countries, the Balkans, the Persian Gulf, the Middle East and Central and South America (Pappas et al., 2006). The most invasive and pathogenic type of human brucellosis is B.melitensis followed by B.abortus and B.suis (Cem Gul and Erdem, 2009).

Infection of total knee or hip arthroplasty by Brucella species is a rare complication with only 19 cases reported in the English literature, furthermore it is only the 4th case described in Israel (Mesner et al., 2007; Joshua et al., 2015).

\section{Case Report}

A 79 year old Jewish Israeli man holds a company that imports meat to Israel from Argentina. He goes to Argentina every couple of months to be present and check the slaughter process. Moreover, he eats the local meat.

The patient was presented to the hospital with the complaint of left knee pain for a period of a year and a half. The pain became worse within the last 3 months before presentation. He is generally healthy without chronic medication intake. No fever, night sweats or weight loss were observed during that period of time. The patient had bilateral total knee arthroplasty, 12 years before presentation in the left knee and 4 years before presentation in the right knee.

In order to relieve his left knee pain he was treated with hot stones in physiotherapy clinic one year before presentation. The stones were applied directly on his knee. The following treatment caused him a local burn that healed with a scar tissue. Later the patient noticed 3 sinus tracts that were draining bloody fatty fluid from and around the scar tissue.

In the clinic blood test showed: CRP: $54.9 \mathrm{mg} \mathrm{L}^{-1}$,

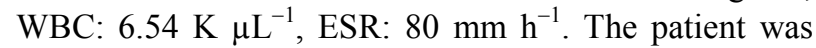
sent for a SPECT-WBC directed at both knees. The test showed an increased uptake in the left knee around the implant. With this test result he went to see the head of the orthopedic arthroplasty department, which decided that the patient must go through a two stage replacement arthroplasty. When the patient was admitted to the hospital for the first stage, we have started an antibiotic regimenwith abroad spectrum coverage that includes: VANCOMYCIN 1 gr b.i.d and CEFTAZIDIME 2 gr t.i.d. He had an X-ray a day before the surgery (Fig. 1a-c).

In the operating room under spinal anesthesia with the use of tourniquet we made a section on his previous scar. Entering the joint space a gush of dark synovial fluid came out. Three swabs and two specimens from the fluid were taken for a Gramm stain and bacterial culture. When the implants were removedwe saw a lack of bone in the tibia and femur with several cysts. We performed a large volume of irrigation with debridement of soft tissue after which we implanted cemented spacer impregnated with GENTAMYCIN 3 gr, CLINDAMYCIN 3 gr and VANCOMYCIN 3 gr. After implantation of cemented spacer we have irrigated again the surgical wound, closed it and left a plastic drain. The sinus tracts were irrigated as well and were closed with sutures. An x ray was taken after the operation (Fig. 2a and b).

For the next two days after the surgery the patient was treated with VANCOMYCIN and CEFTAZIDIME until preliminary results from the cultures arrived. The cultures presented a rose Bengal stain which showed positive for Brucella species with titers of antibodies IgG 1:640 and IgM 1:1920. Our infection disease specialists send those specimens to a veterinary hospital in order to understand which subtype of Brucella we are dealing with.

Meanwhile the patient was released from the hospital with the treatment of GENTAMYCIN $320 \mathrm{mg}$ daily for 3 weeks together with DOXYCYCLINE $100 \mathrm{mg}$ b.i.d and RIFAMPIN $600 \mathrm{mg}$ daily for the duration of three months. Upon his next scheduled meeting, twelve days after his release from hospital, a blood test was taken and showed:

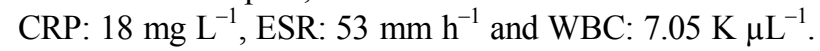




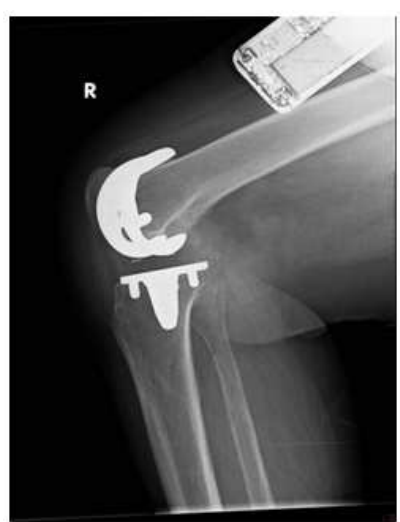

(a)

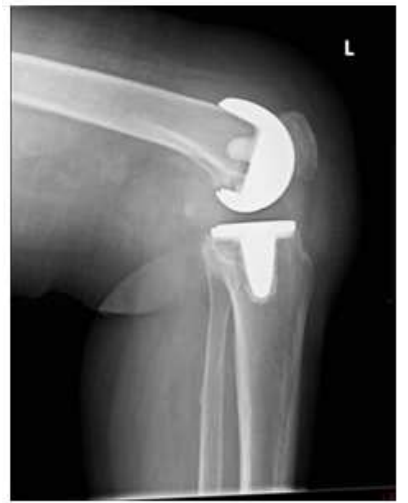

(b)

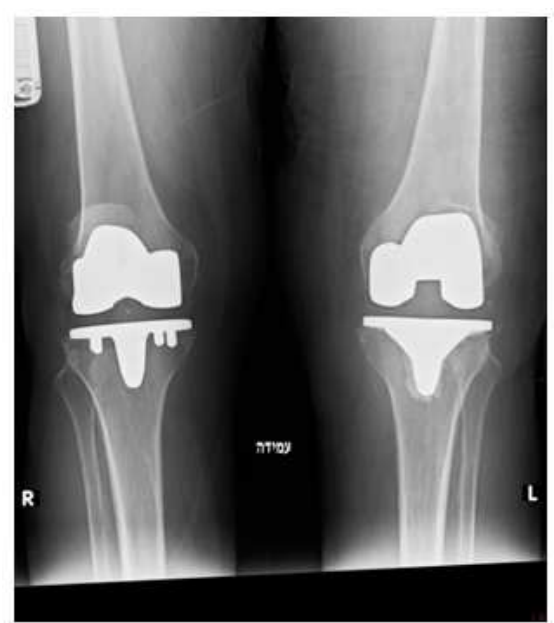

(c)

Fig. 1a-c. Post op X-Ray AP and LAT, right cementless LCS, left cemented SIGMA RP

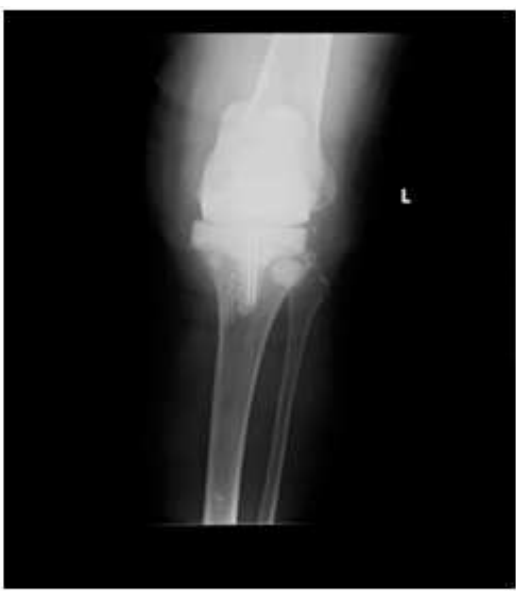

(a)

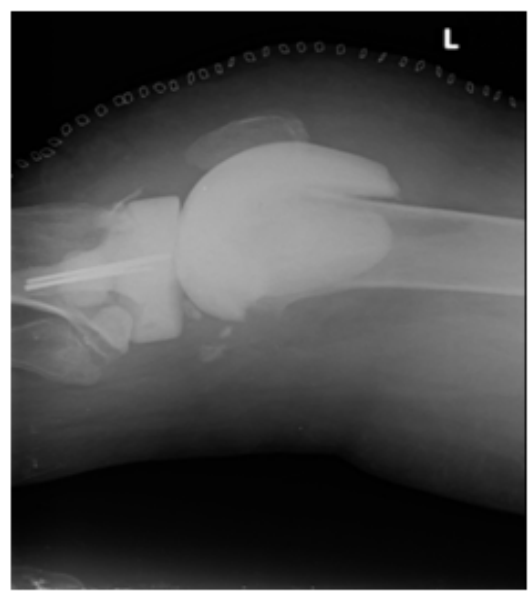

(b)

Fig. 2a-b. Status post 1 st stage of revision with articulating spacer with antibiotics

By that time an answer from the veterinary hospital has arrived and stated that our patient was infected with Brucella melitensis. It was decided that after the third week of treatment with GENTAMYCIN, the patient will continue only with DOXYLCYCLINE and RIFAMPIN for a period of three months. Moreover, he was asked to arrive for CRP and Brucella serology checkup monthly and $\mathrm{CBC}$ test every two weeks. 


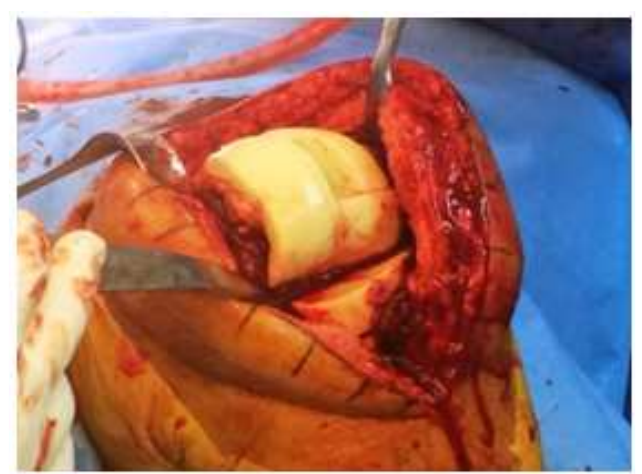

Fig. 3. Intraoperative pictures during stage 2, removal of spacer

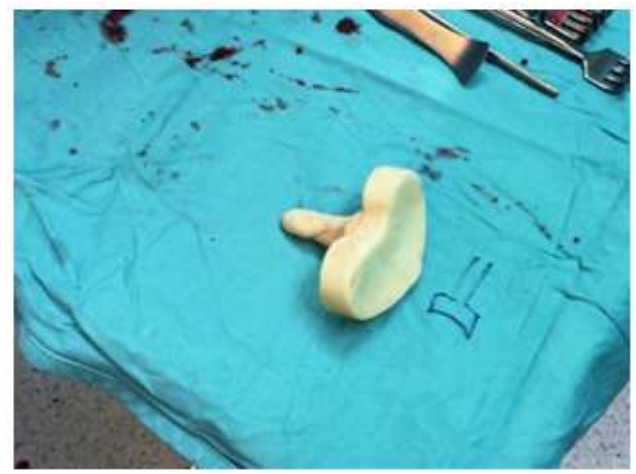

Fig. 4. Tibial spacer with antibiotics

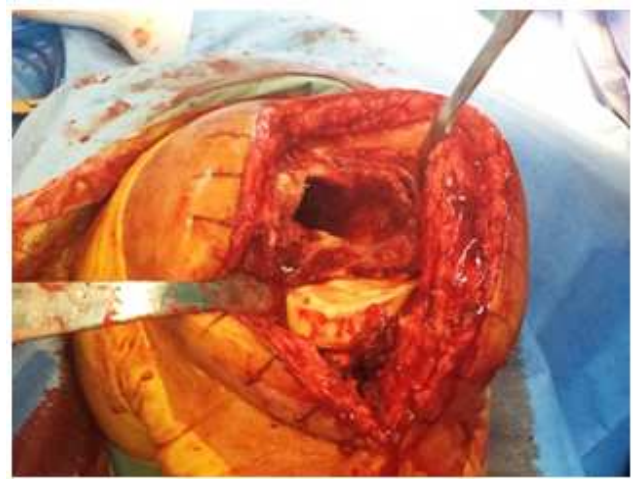

Fig. 5. Severe femoral bone loss after spacer removal

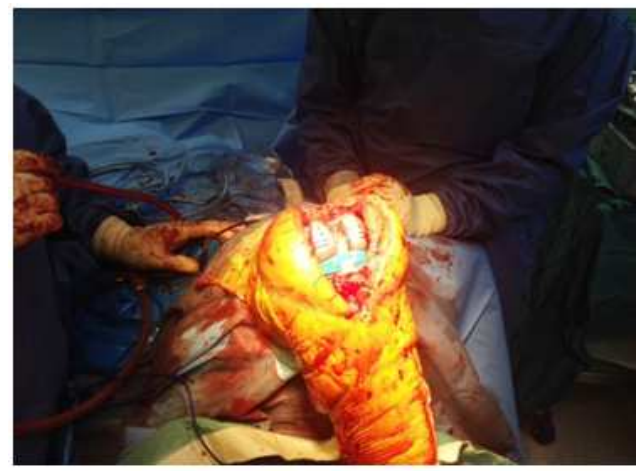

Fig. 6. Stage 2 trial reduction
The next follow up was two weeks later, where CRP:

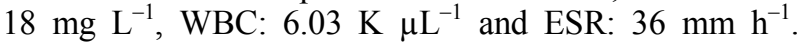
Whenthe patient arrived for his third checkup, 20 days after the previous one, we saw that the lab inflammatory

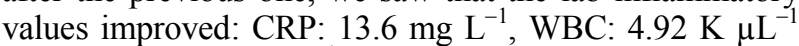
and ESR: $25 \mathrm{~mm} \mathrm{~h}^{-1}$. His serology profile showed: B.melitensis $\mathrm{Ab} \mathrm{1:5++++}$ and B.abortus 1:10++++ complement fixation test, together with B.melitensis $\mathrm{Ab}$ $1: 20++++$ and B.abortus $\mathrm{Ab} 1: 20++++$ serum agglutination test. By examination, his orthopedic surgeon stated that the knee looks good without any swelling or signs of inflammation. The orthopedic team together with the infection disease specialists decided that the infection is under suppression and his inflammation markers are very low, which allowed us to proceedwith stage two of the operation. Three days before the second stage surgery we made another CRP test which was $10.3 \mathrm{mg} \mathrm{L}^{-1}$.

Two months after the first, we performed the second stage operation which included removal of the temporary antibiotic impregnated stem, tissue debridement of fibrotic areas and irrigation, no signs of inflammation or contamination were observed during the surgery. We did observe lack of bone material and lack of stabilizing soft tissue, so it was decided to use a hinged constrained prosthesis with metal augments (Fig. 5-7b).

The wound was closed with suturing of deep tissue, fascia and subcutaneous region. The skin was closed with staples, above which we put special absorbing patch that the patient needs to remove on day 5 after surgery. We did not leave any plastic drain inside the wound. The patient stayed for two days observation in the hospital and was released without any signs of inflammation.

One month after his operation a serology test showed slight increase in B.abortus $\mathrm{Ab} 1: 20++++$ and B.melitensis $\mathrm{Ab} 1: 40++++$. Together with that we checked CRP and WBC levels; $13.4 \mathrm{mg} \mathrm{L}^{-1}$ and $4.69 \mathrm{~K}$ $\mu \mathrm{L}^{-1}$ respectfully. Because of the rise in serology and CRP values infectious disease specialists decided to continue DOXYCYCLINE and RIFAMPIN for another 2 weeks, which sums it to six weeks of treatment since the second operation. Two weeks after the last checkup the patient was seen by his orthopedic surgeon, who stated that the operated wound looks good, the position of the stem is good, the patient is allowed to walk with the help of a stick. The following treatment included DOXYCYCLINE $100 \mathrm{mg}$ daily and analgesics (Fig. 7c).

One month later, after a treatment with DOXYCYCLINE $100 \mathrm{mg}$ per day regimen, the patient complained that his operated lower limb is painful but he could not point on the exact place. The knee was swollen but with no general fever or local heat and no redness. Laboratory tests showed: CRP: $9.9 \mathrm{mg} \mathrm{L}^{-1}$, WBC: 5.12 $\mathrm{K} \mu \mathrm{L}^{-1}$. Because of his complaint and swollen knee and in spite his laboratory values were improving, we have decided to start again treatment with DOXYCYCLINE $100 \mathrm{mg}$ b.i.d and RIFAMPIN $600 \mathrm{mg}$ daily. Another meeting was scheduled in a month and twenty days. 


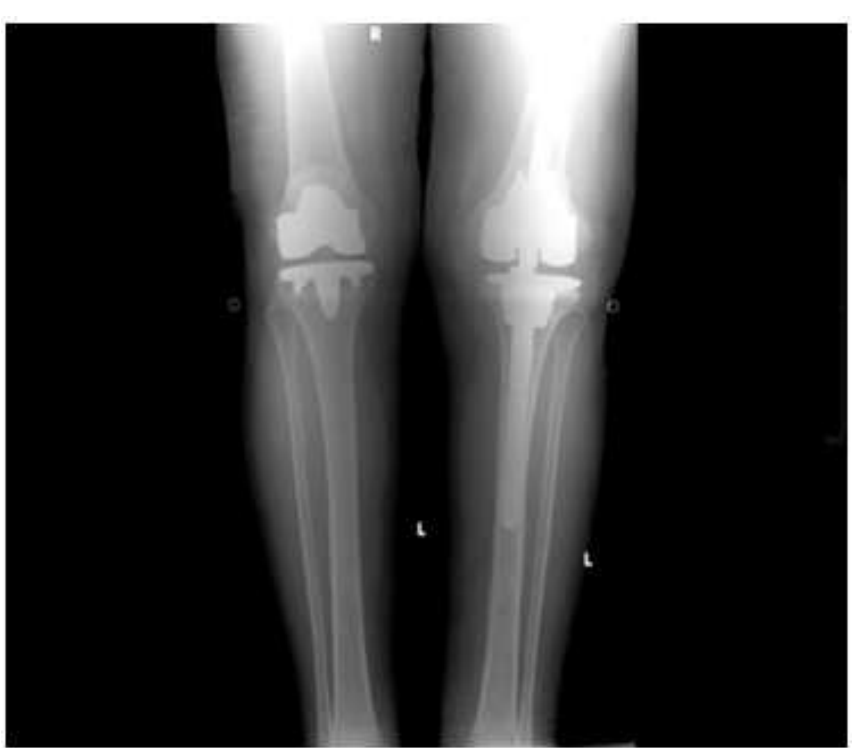

(a)

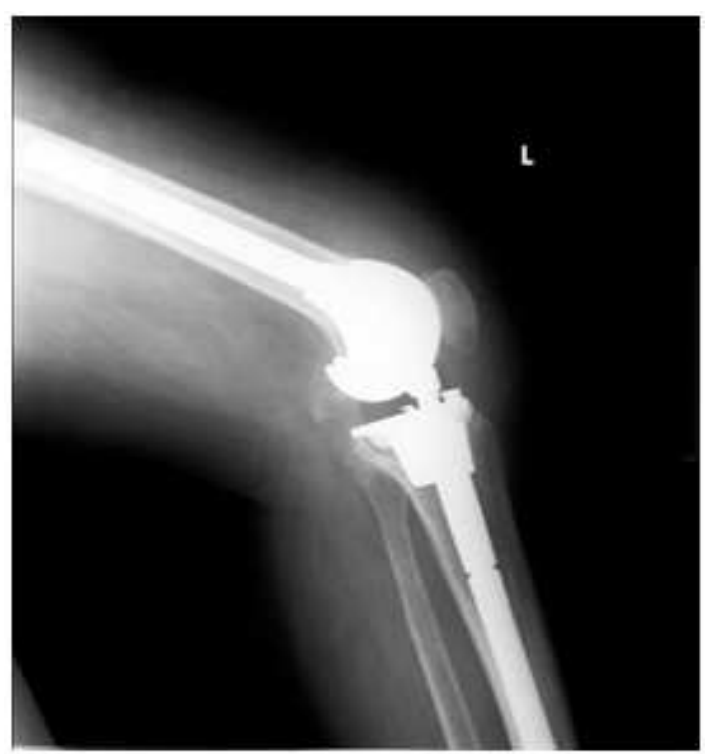

(b)

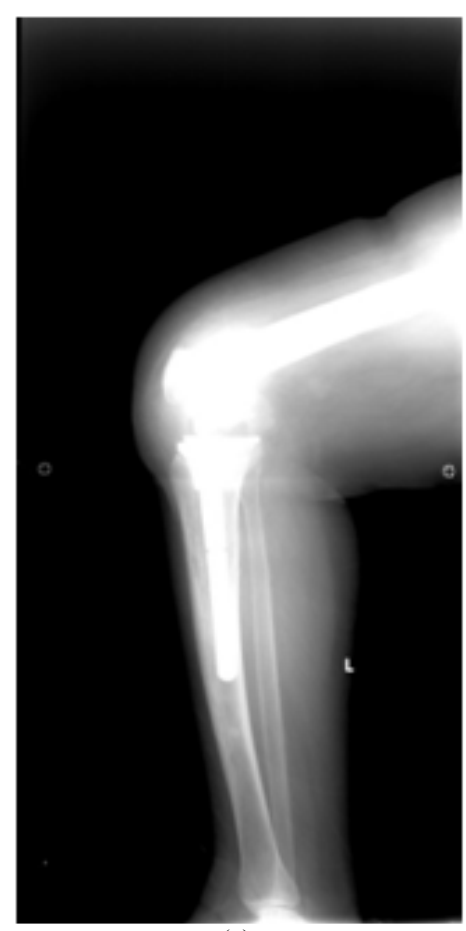

(c)

Fig. 7. Status post 2 nd stage of left total knee revision with long stem intramedullary rods and metaphysial trabecular metal augments

When the patient arrived to his next meeting we had already the results of Brucella serology taken on his last appointment. The results showed Brucella TIT Ab IgM: 1:320 and Ab IgG: 1:320, a positive rose Bengal test. B.melitensis Ab serology 1:20+, B.abortus Ab serology

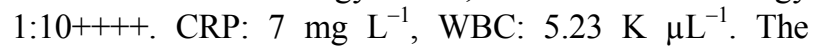
infectious disease specialists decided upon these values that we managed to confine the disease but we are unable to eradicate it completely. We decided to add a third medicine TRIMETHOPRIM-SULFAMETHOXAZOLE $960 \mathrm{mg}$ b.i.d to DOXYCILLIN $100 \mathrm{mg}$ b.i.d and to change the dosage of RIFAMPIN to $300 \mathrm{mg}$ b.i.d for a period of at least half a year.

Under this treatment, the patient is feeling very good, he is able to walk pain free and upon physical examination there are no signs of inflammation or infection. 
The patient is under continuous observation of infectious disease specialists and orthopedic surgeon, but he will probably need a lifetime treatment with antibiotics because of chronic Brucella infection that we are able to suppress but not eradicate.

\section{Discussion}

Brucellosis is a zoonotic disease transmitted to humans through contact with infected animal hosts. Brucellosis can involve any organ in the human body as is known as a "great imitator" (Cem Gul and Erdem, 2009; Franco et al., 2003). Incubation period is usually 14 weeks and the onset of symptoms can be abrupt or insidious. Most frequent complaints are arthralgia, fatigue, undulant fever, malodorous respiration, lack of appetite, myalgia, back pain and is commonly associated with hepatosplenomegaly (Franco et al., 2003; Pappas et al., 2005). We want to emphasize that osteoarticular involvement is the most common complication seen almost in half of brucellosis infections (Cem Gul and Erdem, 2009; Colmenero et al., 1996; Buzgan et al., 2010). The lumbar vertebrae, hips, knees and ankles are the most often involved joints (Cem Gul and Erdem, 2009; Geyik et al., 2002). As we mentioned earlier there were only 19 cases reported worldwide in the English literature and this is the 4th case reported in Israel (Mesner et al., 2007; Joshua et al., 2015). Most of the cases occurred in the Mediterranean region or in the Middle East and only two occured in North America. Although there is a big chance that our patient acquired the infection in South America, we do not have any prove regarding that issue. The patients in cases that were reported in the literature had symptoms that presented from 2 month till 14 years after the primary surgery and their symptoms ranged from 1 month to 2 years before they saw a clinician. Most of the patients either ingested unpasteurized dairy products or worked closely with live stock. Laboratory evaluation revealed elevated ESR, CRP or both in 9 cases. In one of the cases, the ESR was normal but a bone scan was positive. Joint aspiration yielded Brucella species in 9 cases, was negative in 3 and not reported in 5 cases.

In nine cases patients presented loose components, while one case was not reported and the remaining were assumed to be well-fixed. Patients that had a loose stem underwent a revision surgery; six cases had a two stage procedure and two cases underwent only one stage procedure. Seven patients, who were considered to have a well fixed component, including those without description of their component status intraoperativly, were treated only with oral antibioticsfor duration of 6 weeks till 26 month. Among those patients that were treated only with oral antibiotics, in two cases there was failure to eradicate the invading organism and they went through a revision surgery. Other patients who have been treated only with antibiotics were doing well during the time of the follow up that lasted from 8 month to 5 years. There were no reports of transmission to hospital or laboratory personnel in any of the cases or reports of precautions taken to limit exposure of operating rooms or hospital personnel (Mesner et al., 2007; Joshua et al., 2015).

\section{Conclusion}

In order to suspect brucellosis as a periprosthetic infection we need to embrace inexorable history taking especially related to consumption of dairy products and close work with live stock. Furthermore, during physical examination it is important to look at the skin surrounding the prosthetic joint and to look for swelling, redness, warmth and draining sinuses. As we saw in most cases, ESR and CRP are the major laboratory values that show inflammation. A positive bone scan helped to confirm infection, but only a culture taken from the infected joint can really confirm the diagnosis of brucellosis infection. Another important issue is the fact that it is very important to have a full collaboration between orthopedic surgeons and the infectious disease specialists and only collaborative work, in our opinion, can bring to eradication of the infection or its complete suppression. During the review of cases we concluded that in case of loose stem, a surgery is needed to replace it, but there is no consensus and data regarding which one or two stage revision is better. In cases where patients were treated solely with antibiotics, without surgery regarding well fixed stems, 7 out of 9 cases reported successful eradication of the bacteria.

We have reviewed infectious disease literature which summarized the antibiotic treatment (Cem Gul and Erdem, 2009). Most commonly the antibiotic regimen included RIFAMPIN, DOXYCYCLINE and STREPTOMYCIN.

\section{Uncomplicated Brucellosis}

Preferred regimen: Doxycycline- $100 \mathrm{mg}$ PO b.i.d for minimum 6 weeks,

plus

Streptomycin 1g IM daily for 2-3 weeks.

Alternative regimen: Doxycycline $100 \mathrm{mg}$ PO b.i.d for at least 6 weeks, plus Gentamycine $5 \mathrm{mg} \mathrm{kg}^{-1}$ IM daily for 1 week. or Doxycycline 100 mg PO b.i.d for minimum 6 weeks, plus Rifampin 600-900 mg daily for minimum 6 weeks. 


\section{Nurobrucellosis}

Preferred regimen: Ceftriaxone $2 \mathrm{mg}$ IV b.i.d for minimum 1 month,

plus

Doxycycline 100 mg PO b.i.d for 4-5 month,

plus

Rifampin 600-900 mg daily for 4-5 month.

Alternative regimen: Trimethoprim-sulfamethoxazole 160-800 mg b.i.d for 5-6 months,

plus

Doxycycline $100 \mathrm{mg}$ PO b.i.d for 5-6 months,

plus

Rifampin 600-900 mg daily for 5-6 months.

We think that brucellosis should be included in the differential list of pathogens that can cause acute and chronic infections around orthopedic implants especially in patients who live in endemic areas.

\section{Impacts}

- The amount of arthroplasties is increasing constantly worldwide. Medical implants are foreign bodies and increase the risk for infection. People after arthroplasty need to be extra careful being in contact with household animals or their secretions because infection can be transmitted through bruises and laceration on the skin, inhalation of infected aerosol, conjuctival inoculation and they should not eat consume unpasteurized milk and dairy products

- Brucellosis is most frequently encountered worldwide zoonotic disease with osteoarticular involvement being its most common complication

- Treating brucellosis infection with periprosthetic involvement is a very complex procedure and we are not always able to eradicate the infection completely

\section{Funding Information}

The article and all related issues were sponsored solely by the authors.

\section{Author's Contributions}

All authors equally contributed in this work.

\section{Ethics}

We received the permission by the ethics commission in our country stating that this article does not possess any ethical problem.

\section{References}

Anonymous, 1995. NIH consensus conference: Total hip replacement. NIH consensus development panel on total hip replacement. JAMA, 273: 1950-1956. PMID: 7783307
Berbari, E.F., A.D. Hanssen, M.C. Duffy, J.M. Steckelberg and D.M. Ilstrup et al., 1998. Risk Factors for prosthetic joint infection: Case-control study. Clin. Infect. Dis., 27: 1247.

DOI: $10.1086 / 514991$

Bosilkowski, M., M. Dimzova and K. Grozdanowski, 2009. Natural history of brucellosis in an endemic region in different time periods. Acta Clin. Croat., 48: 41-46. PMID: 19623871

Buzgan, T., M.K. Karahocagil, H. Irmak, A.I. Baran and H. Karsen et al., 2010. Clinical manifestations and complications in 1028 cases of brucellosis: A retrospective evaluation and review of the literature. Int. J. Infect. Dis., 14: e469-e478.

DOI: $10.1016 /$ j.ijid.2009.06.031

Cem Gul, H. and H. Erdem, 2009. Brucella Species. In: Mandell, Douglas and Bennett's Principles and Practice of Infectious Diseases: Expert Consult Premium Edition, Bennett, J.E., M.D. Raphael Dolin and M.J. Blaser, Elsevier-Health Sciences Division, ISBN-10: 1455748013, pp: 2584-2588.

Colmenero, J.D., J.M. Reguera, F. Martos, D. Sánchez-deMora and M. Delgado et al., 1996. Complications associated with Brucella melitensis infection: A study of 530 cases. Medicine, 75: 195-211. DOI: 10.1097/00005792-199607000-00003

Doganay, M. and B. Aygen, 2003. Human brucellosis: An overview. Int. J. Infect. Dis., 7: 173-182. DOI: 10.1016/S1201-9712(03)90049-X

Elie, B. and M.B. Larry, 2015. Clinical manifestations and diagnosis of prosthetic joint infections. UpToDate, Inc.

Franco, M.P., M. Mulder and R.H. Gilman, 2003. Human brucellosis. Lancet Infect. Dis., 7: 173-182.

Geyik, M.F., A. Gur, K. Nas, R. Cevik and J. Saraç et al., 2002. Musculoskeletal involvement of brucellosis in different age groups: A study of 195 cases. Swiss Med. Wkly, 132: 98-105. PMID: 11971204

Jamsen, E., P. Nevalainen, A. Eskelinen, K. Huotari and J. Kalliovalkama et al., 2012. Obesity, diabetes and preoperative hyperglycemia as predictor of periprosthetic joint infection: A single-center analysis of 7181 primary hip and knee replacement for osteoarthritis. J. Bone Joint Surg. Am., 94: e101-e101. PMID: 22810408

Joshua, T., M.D. Carothers, C. Megin, D.V.M. Nichols and M.P.H. Deborah et al., 2015. Failure of total hip arthroplasty secondary to infection caused by Brucella abortus and the risk of transmission to operative staff. Am. J. Orthop., 44: E42-E45. PMID: 25658081

Kurtz, S., K. Ong, E. Lau, D. Berry and J. Parvizi, 2010. Prosthetic joint infection risk after TKA in the Medicare population. Clin. Orthop. Relat. Res., 468: 52-56. DOI: 10.1007/s11999-009-1013-5 
Mesner, O., K. Riesenberg, N. Biliar, E. Borstein and L. Bouhnik et al., 2007. The many faces of humanto-human transmission of brucellosis: Congenital infection and outbreak of nosocomial disease related to an unrecognized clinical case. Clin. Infect. Dis., 45: e135-e140. DOI: 10.1086/523726

Pappas, G., N. Akritidis, M. Bolkowski and E. Tsianos, 2005. Brucellosis. N. Eng. J. Med., 352: 2325-2336. DOI: 10.1056/NEJMra050570

Pappas, G., P. Papadimitriou, N. Akritidis, L. Christou and E.V Tsianos, 2006. The new global map of human brucellosis. Lancet Infect. Dis., 6: 91-99. DOI: 10.1016/S1473-3099(06)70382-6
Poss, R., T.S. Thornhill, F.C. Ewald, W.H. Thomas and N.J. Batte et al., 1995. Factors influencing the incidence and outcome of infection following total joint arthroplasty. Clin. Orthop. Relat. Res., 55: 117-216. PMID: 6692605

Singh, K., 2009. Laboratory-acquired infections. Clin. Infect. Dis., 49: 142-147. DOI: 10.1086/599104

Widmer, A.F., 2001. New developments in diagnosis and treatment of infection in orthopedic implants. Clin. Infect. Dis., 33: S94-S106. DOI: 10.1086/321863

Zimmerli, W., A. Trampuz and P.E. Ochsner, 2004. Prosthetic-joint infections. N. Engl. J. Med., 350: 1422-1429. DOI: 10.1056/NEJMra040181 